\title{
Erratum to: The Positive Effects of Exercise in Chemotherapy-Related Cardiomyopathy
}

Elena Cavarretta, Giorgio Mastroiacovo, Annik Lupieri, Giacomo Frati, and Mariangela Peruzzi

Erratum to:

Chapter 8 in: J. Xiao (ed.), Exercise for Cardiovascular

Disease Prevention and Treatment, Advances in Experimental Medicine and Biology 1000, https://doi.org/10.1007/978-981-10-4304-8_8

The original volume was published with the authors' names inadvertently interchanged. This has been corrected in this updated volume.

The updated online version of this chapter can be found at https://doi.org/10.1007/978-981-10-4304-8_8 